\title{
Attosecond tunnelling interferometry
}

\author{
O. Pedatzur ${ }^{1 \star}$, G. Orenstein ${ }^{1}$, V. Serbinenko², H. Soifer1, B. D. Bruner ${ }^{1}$, A. J. Uzan ${ }^{1}$, D. S. Brambila ${ }^{2}$, \\ A. G. Harvey ${ }^{2}$, L. Torlina ${ }^{2}$, F. Morales ${ }^{2}$, O. Smirnova $^{2 \star}$ and N. Dudovich ${ }^{1 \star}$
}

\begin{abstract}
Attosecond physics offers new insights into ultrafast quantum phenomena involving electron dynamics on the fastest measurable timescales. The rapid progress in this field enables us to re-visit one of the most fundamental strong-field phenomena: field-induced tunnel ionization ${ }^{1-3}$. In this work, we employ high-harmonic generation to probe the electron wavefunction during field-induced tunnelling through a potential barrier. By using a combination of strong and weak driving laser fields, we modulate the atomic potential barrier on optical subcycle timescales. This induces a temporal interferometer between attosecond bursts originating from consecutive laser half-cycles. Our study provides direct insight into the basic properties of field-induced tunnelling, following the evolution of the electronic wavefunction within a temporal window of approximately $\mathbf{2 0 0}$ attoseconds.
\end{abstract}

Quantum tunnelling has been a subject of numerous studies triggering deep theoretical questions, some of which, related to the duration of the tunnelling process, are still hotly discussed ${ }^{4,5}$. When tunnelling is induced by a strong laser field ${ }^{6}$, the barrier oscillates instead of being static. Its basic properties are subject to the rapid subcycle modification of the electromagnetic field, having a natural timescale in the attosecond (as) regime. Within a small fraction of the optical cycle both the width and the height of the tunnelling barrier increase (Fig. 1a), leading to an exponential fall of the tunnelling probability and a significant shift of the exit point. This rapid modification of the barrier raises fundamental questions that are at the heart of strong-field physics-does a liberated electron carry any memory regarding its tunnelling experience? Can we resolve the fingerprint of traversing a rapidly modulated barrier on a liberated electron? In this paper we provide a unique insight into the subtle details of field-induced tunnelling - the starting point for a range of strong-field phenomena ${ }^{7-9}$. By taking a series of snapshots we are able to follow how the instantaneous tunnelling probability changes within a temporal window of 200 as. Most importantly, we identify a clear signature of the non-quasi-static nature of the tunnel ionization process, leaving its fingerprint on both the amplitude and the phase of the electronic wavefunction.

Recent progress in attosecond science has led to several important breakthroughs, shedding new light on the field-induced tunnelling process. Pioneering experiments have measured the tunnelling ionization yield ${ }^{1}$, the validity of tunnelling models ${ }^{10}$, the attosecond dynamics associated with optical tunnelling ${ }^{2}$, the moment at which the electron leaves the tunnelling barrier ${ }^{3,11}$ and simultaneous excitation of the parent ion during tunnel ionization $^{12,13}$. These complementary approaches share a common property-the electron is probed after it leaves the tunnelling barrier. Probing the electron during tunnelling requires an accurate manipulation of the barrier itself. Inspired by the works of Büttiker and Landauer ${ }^{14}$, we manipulate the spatiotemporal shape of the oscillating barrier and record its signature on the tunnelling electron's wavepacket.

Our measurement scheme is composed of two fundamental components. First, probing the tunnelling process without changing the basic mechanism requires a perturbative manipulation of the barrier. We apply such a probe via the addition of a weak second-harmonic (SH) field in parallel to the strong fundamental field ${ }^{15}$ modifying the structure of the barrier on a $1 \%$ level. The second component, associated with the readout, takes advantage of an interferometric measurement ${ }^{16}$, inherent in our high-harmonic generation (HHG) experiment, which maps the subtle modifications of the electronic wavefunction into a clear experimental observable.

Consider a Young's two-slit interferometer in which an electronic wavefunction interacts with two potential barriers placed in each of the slits, as in Fig. 1b. The interference pattern is dictated by the complex phase accumulated by the electron as it penetrates through each of the barriers, as well as the difference due to free propagation. A minuscule difference between the two barriers, leading to a small complex phase difference, $\sigma$, between the two parts of the wavefunction, will be mapped by the interferometer to a substantial modification of the diffraction pattern. Whereas the real part of this differential phase leads to a lateral shift of the interference pattern, its imaginary part modifies the fringe contrast.

We induce a temporal analogue of this interferometer via a HHG measurement (Fig. 1c). An intense infrared pulse bends the binding atomic potential, leading to tunnel ionization. The oscillatory laser field then drives the liberated electron back to the parent ion, leading to recollision, followed by the emission of an attosecond pulse ${ }^{17}$. The pulses emitted every laser half-cycle interfere in the spectral domain, generating a spectral comb of harmonics in the extreme ultraviolet (XUV) regime. This process, induced via two consecutive half-cycles, defines the temporal interferometer. Resolving two neighbouring (odd and even) harmonics is equivalent to the detection of a single fringe in the interference pattern.

When the process is driven by a single-colour field the two slits are identical and the interference pattern includes only odd harmonics. We break this symmetry by adding a weak $\mathrm{SH}$ field, polarized parallel to the strong fundamental field. The total field interacting with the electron is the temporal derivative of the vector potential $A(t)=A_{0}(\sin (\omega t)+\varepsilon \sin (2 \omega t+\phi))$, where $\phi$ and $\varepsilon$ are the phase delay and amplitude ratio between the $\mathrm{SH}$ and fundamental fields, respectively. Such symmetry breaking has already been observed and treated in the classical regime ${ }^{15}$. The additional $\mathrm{SH}$ field slightly enhances the total electric field during ionization in one half-cycle, whereas in the subsequent half-cycle it is reduced. This gives rise to a small difference between the two barriers as well as between the two trajectories in the continuum. The complex phase difference between the two paths, $\sigma$, modifies the interference

${ }^{1}$ Department of Physics of Complex Systems, Weizmann Institute of Science, 76100 Rehovot, Israel. ${ }^{2}$ Max Born Institute, Max-Born-Strasse 2A,

D-12489 Berlin, Germany. *e-mail: orenpedatzur@gmail.com; smirnova@mbi-berlin.de; nirit.dudovich@weizmann.ac.il 
a

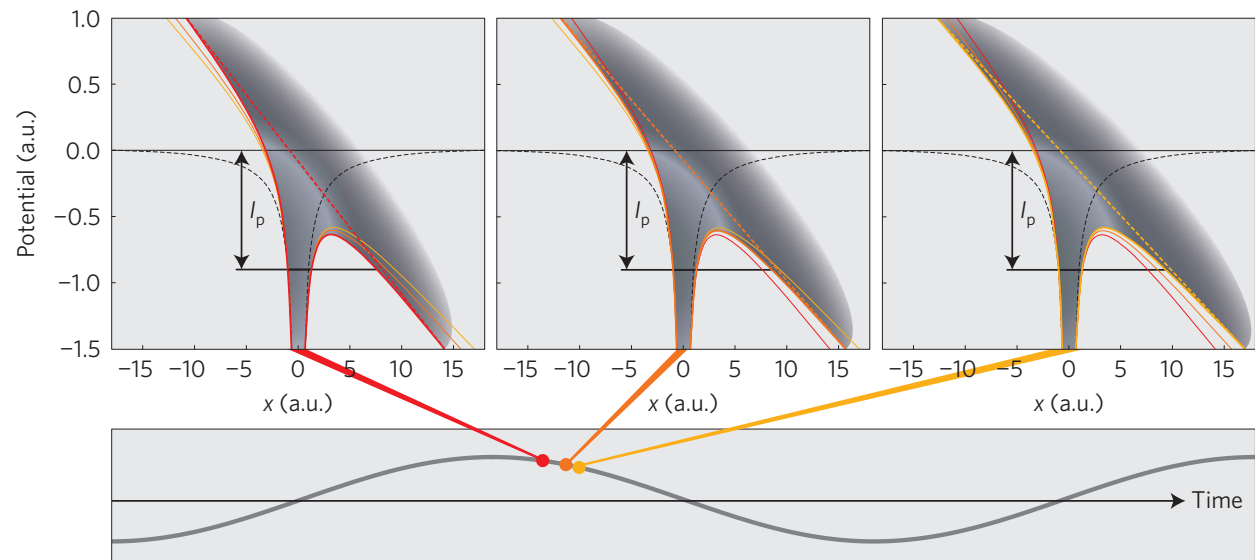

b
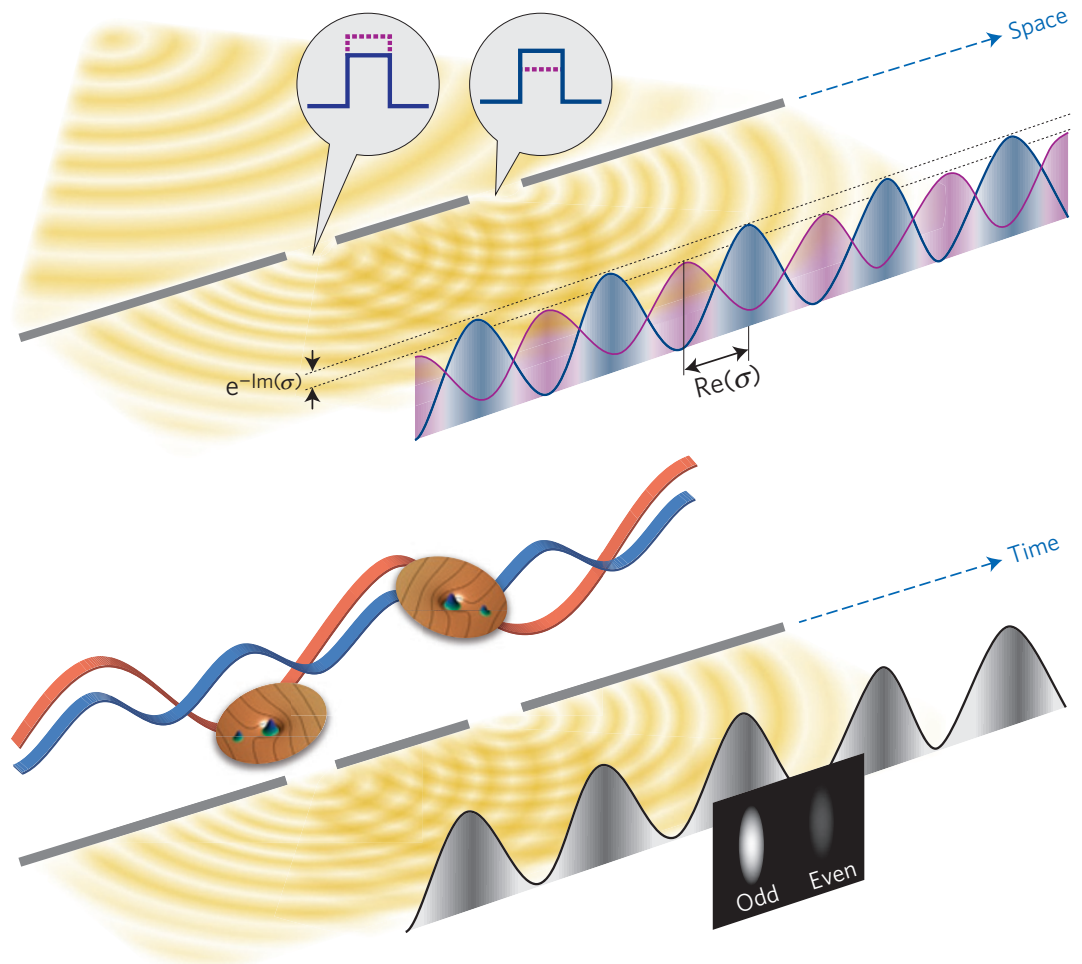

Figure 1 | Interferometry of tunnelling electrons. a, The time-dependent barrier created by the Coulomb potential and the oscillating laser field changes

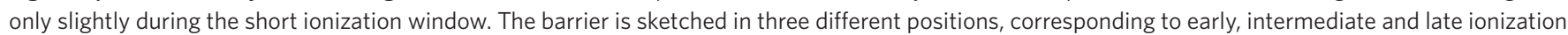

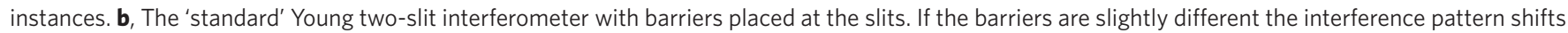

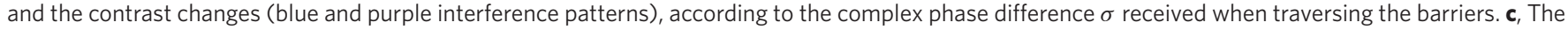
temporal equivalent of the 'barriered' Young interferometer: interference between the signals emitted from two consecutive optical half-cycles. When driven by a monochromatic strong laser, the oscillating field bends the atomic potential identically in consecutive half-cycles, giving rise to identical ionization events and the appearance of only odd harmonics. By adding a weak SH field the symmetry between the consecutive half-cycles is broken (the barriers are slightly different for the two ionization events), such that the interference pattern shifts and its contrast changes, allowing the appearance of even harmonics.

pattern in the spectral domain, leading to the generation of both even and odd harmonics. Their relative signal can be expressed $\mathrm{as}^{18}$ :

$$
\begin{array}{r}
I_{N}=I_{0}^{(N)}\left\{\begin{aligned}
|\cos (\varepsilon \sigma(N, \phi))|^{2}, & N \text { odd } \\
|\sin (\varepsilon \sigma(N, \phi))|^{2}, & N \text { even }
\end{aligned} \Rightarrow\right. \\
\operatorname{Re}(\sigma(N, \phi))=\frac{1}{2} \cos ^{-1}\left(\frac{I_{\text {diff }}^{(N)}(\phi)}{I_{0}^{(N)}}\right) \\
\operatorname{Im}(\sigma(N, \phi))=\frac{1}{2} \cosh ^{-1}\left(\frac{I_{\text {sum }}^{(N)}(\phi)}{I_{0}^{(N)}}\right)
\end{array}
$$

The total fringe intensity, $I_{0}^{(N)}$, is a slowly varying function of the harmonic order $N$, and is taken to be the same for adjacent harmonics. Calculating the sum $\left(I_{\text {sum }}^{(N)}\right)$ and difference $\left(I_{\text {diff }}^{(N)}\right)$ of adjacent odd and even harmonics, as in the right side of equation (1) enables us to directly extract the complex value of $\sigma$.

In our experiment we have realized this scheme, producing HHG in He using a strong infrared field and a parallel and collinear perturbative $\mathrm{SH}$ field (see Methods). We have recorded and analysed the harmonics' modulations with the two-colour delay, $\phi$, over a spectral range spanning from the threshold up to the cutoff harmonic. Experimentally, the two-slit picture is averaged over both time (owing to the multicycle nature of the fundamental field) and 

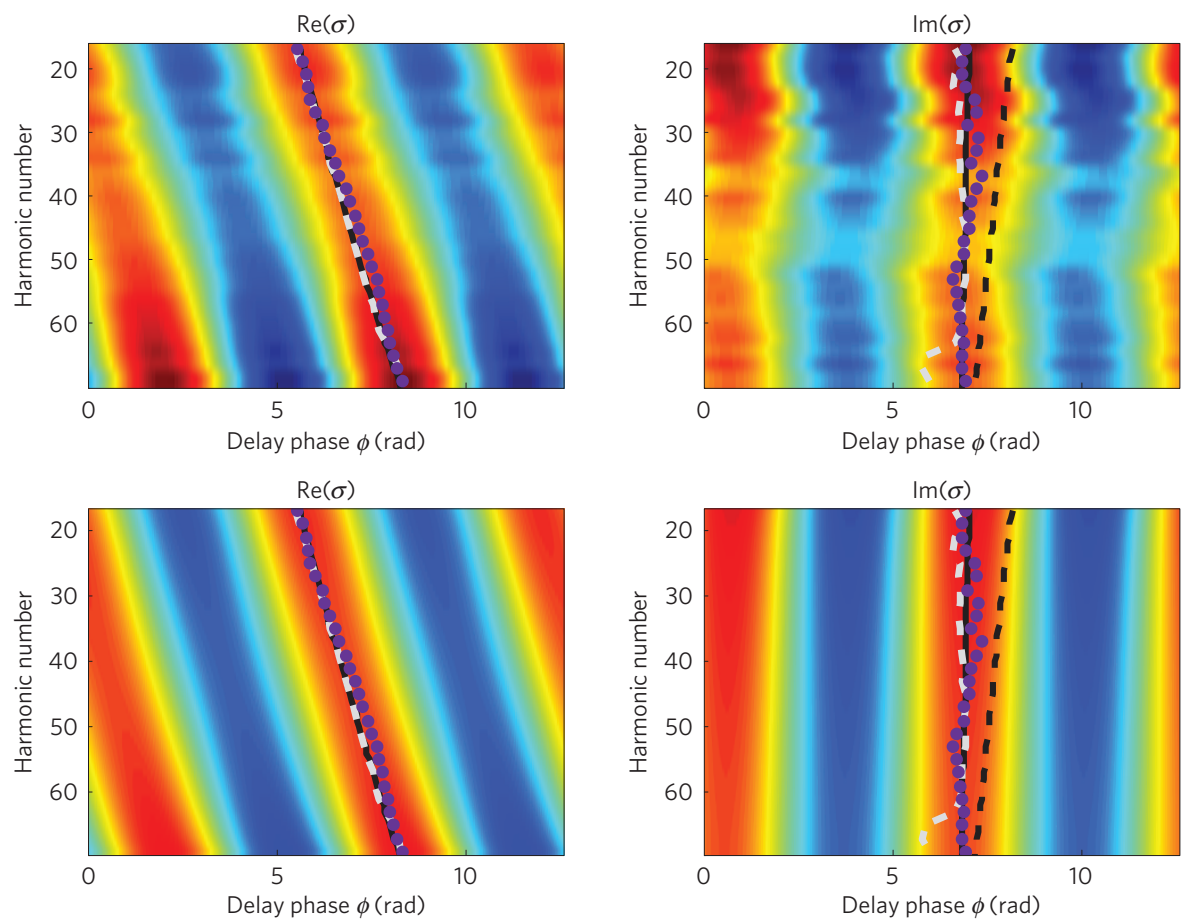

Figure 2 | Interference phase. $\sigma(N, \phi)$, the complex phase difference between paths taken on consecutive half-cycles in He, as a function of the two-colour delay phase and harmonic number. The top row gives the experimental results and the bottom row gives the theoretical calculations involving the SPA and neglecting the core potential. The experimental phase delay that maximizes $\operatorname{Re}(\sigma)$ and $\operatorname{Im}(\sigma)$ is marked by purple dots. Clearly, there is a good agreement between theory and experiment for both real (left column) and imaginary (right column) parts, although the imaginary part is more sensitive to noise. The black solid lines represent the maximal values of the real and imaginary components of $\sigma(N, \phi)$ according to the SPA. The dashed black line depicts the maximal values of $\operatorname{Im}(\sigma)$ according to the quasi-static approximation. The maximal values of $\operatorname{Re}(\sigma)$ and $\operatorname{Im}(\sigma)$ extracted from ab initio numerical solution of time-dependent Schrödinger equation for a He atom (see Supplementary Information) are represented by the grey dashed line.

space (owing to the spatial distribution of the interaction). However, the high nonlinearity associated with the HHG mechanism acts as a spatiotemporal filter, where only high-intensity cycles contribute. Residual averaging is eliminated by a careful spatiospectral analysis. A systematic study, described in the Supplementary Information, confirms that our experiment can indeed be described via a twoslit picture.

Figure 2 describes the measurement of $\operatorname{Re}(\sigma(N, \phi))$ and $\operatorname{Im}(\sigma(N, \phi))$ (upper panels), demonstrating the ability to decouple the imaginary component that is usually associated with the tunnelling process, from the real component that is associated with the free propagation of the electron. Note that the delay phase, $\phi$, is known up to a constant. We calibrate this global phase by shifting the calculated data to the point of maximal cross-correlation with the experimental data (Supplementary Information).

A description of the quasi-static regime of strong-field ionization was first introduced by Keldysh ${ }^{6}$. In this regime, the electron tunnels through a static barrier, dictated by the instantaneous value of the laser field. According to this picture, for a given trajectory, the $\mathrm{SH}$ field will maximize the ionization probability when its peak synchronizes with the ionization time, maximizing $|\operatorname{Im}(\sigma)|$. This gives rise to a simple mapping for each trajectory connecting the time of ionization $t_{0}$, to the delay $\phi$ which satisfies such synchronization. The quasi-static picture is justified in the limit of a small Keldysh parameter $\gamma \equiv \sqrt{I_{\mathrm{p}} / 2 U_{\mathrm{p}}} \ll 1$, where $I_{\mathrm{p}}$ is the ionization potential and $U_{\mathrm{p}}$ is the ponderomotive energy of the electron in the laser field (Supplementary Information). The black dashed line in Fig. 2 describes the prediction of the quasi-static theory, showing a deviation from the experimental results. Clearly, field-induced tunnel ionization in our experimental conditions $(\gamma=0.68)$ does not follow the instantaneous value of the laser field as would be expected in the quasi-static limit.
A deeper insight is obtained by considering strong-field light-matter interaction beyond the quasi-static approximation. The spectrum emitted in a single recollision event is dictated by the coherent addition of all possible quantum paths that contribute to the process. These interfere with a relative phase $S\left(t_{0}, t_{1}, p\right)$, known as the semi-classical action, which depends on the electron's instant of birth $t_{0}$, instant of return $t_{1}$ and canonical momentum $p$. For sufficiently high harmonic number the interaction is dominated by the stationary points of $S\left(t_{0}, t_{1}, p\right)$ (refs 19,20). In this case the stationary phase approximation (SPA) can be applied, naturally invoking the language of quantum trajectories. According to the SPA, each harmonic order $N$ is dominated by the electron trajectory associated with the stationary ionization time $t_{0, \mathrm{st}}^{(N)}$, recollision time $t_{1, \mathrm{st}}^{(N)}$ and momentum $p_{\mathrm{st}}^{(N)}$, which are complex, reflecting the quantum nature of the phenomena. The real part of the ionization time $\operatorname{Re}\left(t_{0, \mathrm{st}}^{(N)}\right)$ is associated with the time at which the electron leaves the barrier, whereas the imaginary part $\operatorname{Im}\left(t_{0, \mathrm{st}}^{(N)}\right)$ is strongly related to the instantaneous tunnelling amplitude (Supplementary Information).

In the case of a sinusoidal driving field, two branches of solutions exist. These are termed 'short' and 'long' trajectories, as they differ in the duration spent by the electron in the continuum. In our analysis we consider only short trajectories, and accordingly in the experiment the contributions of long trajectories are filtered out by taking advantage of the different phase-matching conditions for the two branches. Returning to our perturbative measurement, the analytic expression for $\sigma$ can be derived by expanding the semi-classical action to first order in $\varepsilon$ (Supplementary Information). Figure 2 exhibits good agreement between our experiment and quantum path analysis (SPA). Note that the spectral range measured in our experiment overlaps with the spectral position of doubly excited resonances in He. However, owing to the short duration of the interaction, imposed by its 
$\operatorname{Re}\left(t_{0, \mathrm{st}}^{(N)}\right) / T_{\text {cycle }}$

a

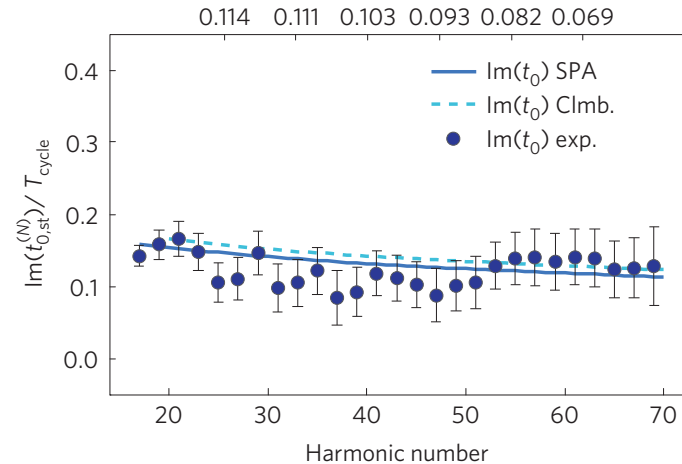

b

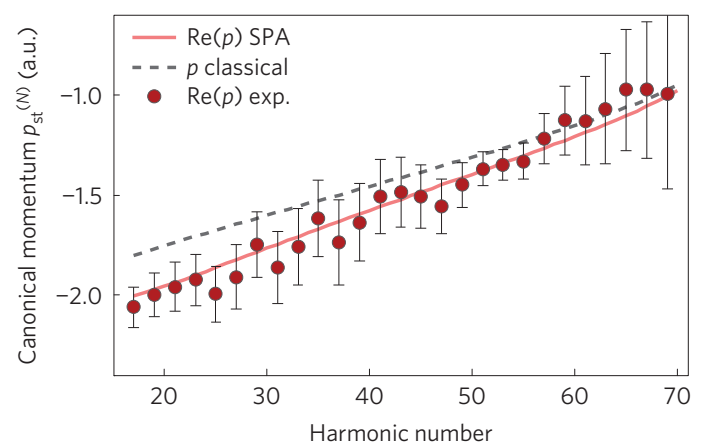

c

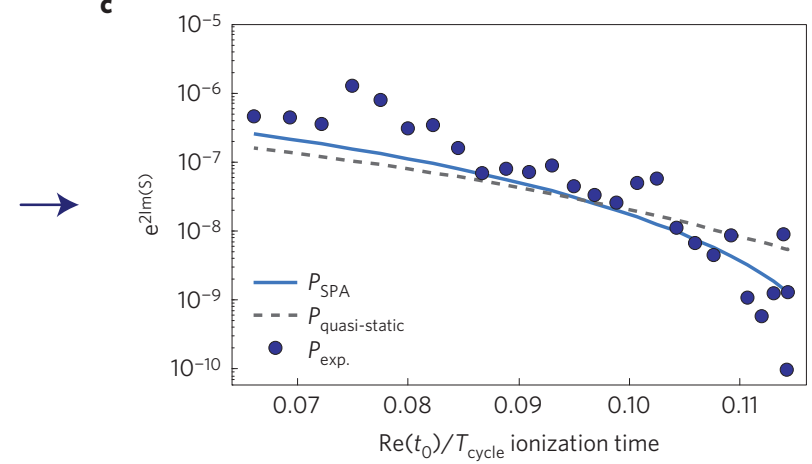

d

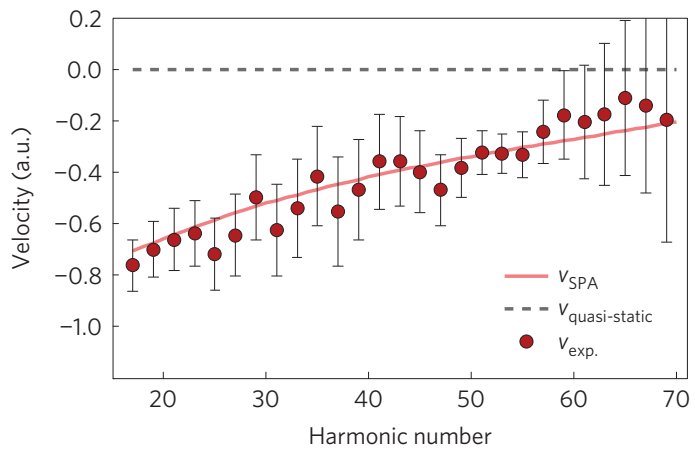

Figure $\mathbf{3}$ | The reconstruction of stationary parameters. a, The imaginary part of the stationary ionization time, Im( $\left.t_{0, s t}\right)$, (blue dots) compared to theoretical SPA values (blue line) and Coulomb corrected (ARM) values. The top axis indicates the corresponding real part of the stationary ionization time. The curve features an increasing slope of $\operatorname{Im}\left(t_{0, s t}\right)$ as a function of $\operatorname{Re}\left(t_{0, s t}\right)$, expressing the different instantaneous height and width of the barrier. b. The real part of the stationary momentum Re $\left(p_{s t}\right)$, (red dots) compared to the SPA (solid red line). A clear shift between the experimental values and a classical analysis (dashed line) is observed, suggesting a momentum compensation due to interaction with the barrier. c, Using the experimental Im( $t_{0, s t}$ ) and $\operatorname{Re}\left(p_{s t}\right)$ and neglecting $\operatorname{Im}\left(p_{s t}\right)$, we retrieve the subcycle time-dependence of the ionization probability (blue dots) and compare it to known values for photo-electrons in the quasi-static barrier approximation (grey dashed curve) and the SPA (blue curve). The instantaneous ionization probability for photo-electrons and HHG agree at early ionization times. The decreasing slope demonstrates the subcycle dependence of the ionization probability on the changing barrier. $\mathbf{d}$, The canonical momentum reveals the initial velocity of the electron in the continuum. The reconstructed values (red dots) are compared to quasi-static $v=0$ expected velocities (dashed grey line) and the SPA prediction (solid red line). The error bars are estimated by propagating the $95 \%$ fit confidence intervals of the phase and amplitude of individual harmonic oscillations (Supplementary Information). The arrows indicate the application of the retrieved stationary parameters to the calculation of the ionization probability and the velocity.

macroscopic properties, no traces of these resonances appear in our spectrum (Supplementary Information). Finally, our SPA analysis neglects the contribution of the Coulomb force on $\sigma$. A careful numerical analysis shows that such corrections are negligible in the conditions of our experiment, as illustrated by the dashed grey line in Fig. 2 (Supplementary Information).

The two key components of our experiment, the perturbative approach and the interferometric measurement, enable us to take an additional important step and reconstruct the main parameters that describe the tunnelling process. Extracting $\sigma(N, \phi)$ for each harmonic number and comparing it to the analytic expression evaluated at the stationary points

$$
\sigma(N, \phi)=\sigma\left(t_{0, \mathrm{st}}^{(N)}, t_{1, \mathrm{st}}^{(N)}, p_{\mathrm{st}}^{(N)}, \phi\right)
$$

provides us with four independent equations defined by the phase and contrast of the oscillations in the real and imaginary parts of $\sigma(N, \phi)$. Earlier studies, ${ }^{3,11,15,21-23}$ have confirmed that $\operatorname{Re}\left(t_{0, \mathrm{st}}^{(N)}\right), \operatorname{Re}\left(t_{1, \mathrm{st}}^{(N)}\right)$ agree with the predicted stationary solutions. On the basis of these studies and the above mapping, it is possible to retrieve the missing parts of the puzzle-the imaginary parts of $t_{0, \mathrm{st}}^{(N)}$ and $t_{1, \mathrm{st}}^{(N)}$ and the complex value of the stationary momentum, $p_{\mathrm{st}}^{(N)}$ (Supplementary Information). The reconstruction procedure yields robust values for $\operatorname{Im}\left(t_{0, \mathrm{st}}^{(N)}\right)$ and $\operatorname{Re}\left(p_{\mathrm{st}}^{(N)}\right)$, but is not sensitive enough to determine $\operatorname{Im}\left(t_{1, \mathrm{st}}^{(N)}\right)$ and $\operatorname{Im}\left(p_{\mathrm{st}}^{(N)}\right)$ within the experimental error.

The reconstruction of $\operatorname{Im}\left(t_{0}\right)$ (Fig. 3a), agrees with the SPA and exhibits a negative slope with the harmonic order. On the basis of the confirmed values of $\operatorname{Re}\left(t_{0, \mathrm{st}}\right)$, we map the instant of birth of each electron trajectory to $\operatorname{Im}\left(t_{0, \mathrm{st}}\right)$ (top axis). The error bars represent the accuracy of the measurement and the reconstruction process (Supplementary Information). Indeed, as time progresses within the optical cycle the instantaneous value of the electric field decreases, leading to an increase of the barrier height and width (Fig. 1a). The dashed blue line describes $\operatorname{Im}\left(t_{0}\right)$ including Coulomb corrections, based on the Analytical R-matrix theory (ARM; ref. 24). As can be clearly observed, these corrections are subtle and are well within our experimental error bars. In the following step we illustrate the outcome of this measurement by calculating the instantaneous ionization probability, using the imaginary part of the action evaluated between the stationary ionization time and its real part, $\exp \left[2 \operatorname{Im}\left(S\left(t_{0, \mathrm{st}}, \operatorname{Re}\left(p_{\mathrm{st}}\right)\right)\right)\right]$. Figure $3 \mathrm{c}$ presents the evolution of the instantaneous tunnelling probability during the ionization window, based on the reconstruction of $\operatorname{Im}\left(t_{0, \mathrm{st}}\right)$, showing the expected rapid fall of the tunnelling probability within a temporal window of 160 as.

The reconstruction of $\operatorname{Re}\left(p_{\text {st }}\right)$ (Fig. $3 b$ ) shows clear deviation from the quasi-static predictions and an excellent agreement with the SPA. What is the origin of this deviation? A fundamental 
assumption of the quasi-static picture is that the electron begins its journey in the continuum with zero velocity ${ }^{6,25}$ (Supplementary Information). The quantum analysis suggests that the electron exits the tunnelling barrier with an initial non-zero velocity, leading to a shift in the canonical momentum. We illustrate this deviation by mapping the reconstructed momentum to the initial velocity in Fig. 3d. According to the SPA, the canonical momentum relates the velocity $v$ and the vector potential $A(t)$ as follows: $\operatorname{Re}\left(v\left(\operatorname{Re}\left(t_{0, \mathrm{st}}\right)\right)\right)=\left(\operatorname{Re}\left(p_{\mathrm{st}}\right)-e A\left(\operatorname{Re}\left(t_{0, \mathrm{st}}\right)\right)\right) / m$. The dashed grey line at $v=0$ marks the static limit and the solid red line describes the initial velocity predicted by the SPA, showing a good agreement with our experimental results. Our ability to probe the electronic wavefunction along its propagation direction enables us to observe a clear signature of the non-static nature of the tunnelling mechanism.

Quantum mechanically, the concepts of measuring both the coordinate and the momentum (or the energy and time of emission) are constrained by the uncertainty principles. However, for very large values of the action, with rapidly changing electron momentum and energy, the relative uncertainty is small. For example, the uncertainty in defining the times we measure is related to the number of harmonics emitted within about laser half-cycle, and is roughly $1 / 70$ of the laser period in our case. Experimentally we resolve the centre of this window with substantially higher accuracy, limited only by the signal-to-noise ratio (Supplementary Information).

To conclude, in this study we demonstrate the ability to probe an electronic wavefunction as it propagates under a field-induced tunnelling barrier. Our measurement reveals a clear signature of the non-adiabatic properties of field-induced tunnelling and its effect on the electronic wavefunction. As we advance to more complex systems, in which non-adiabatic, multi-electron phenomena are expected to be observed ${ }^{26-29}$, our approach will become an essential component. In many molecular systems the tunnelling process involves rapid multi-electron dynamics associated with a coherent population of multiple electronic states. The evolution of this initial excitation on the attosecond timescale may lead to such important phenomena as sub-femtosecond charge migration and charge-directed reactivity ${ }^{30}$. The interaction between the outgoing electron and the electronically excited core during ionization should leave its mark on ionization times and subcycle ionization amplitudes $^{29}$. Our approach to measuring these quantities with unprecedented temporal accuracy provides a new tool for detecting and resolving these multi-electron phenomena. Such observations hold the potential of revealing yet unobserved interactions which are at the forefront of ultrafast science.

\section{Methods}

Methods and any associated references are available in the online version of the paper.

Received 15 June 2014; accepted 14 July 2015; published online 24 August 2015

\section{References}

1. Uiberacker, M. et al. Attosecond real-time observation of electron tunnelling in atoms. Nature 446, 627-632 (2007).

2. Eckle, P. et al. Attosecond ionization and tunneling delay time measurements in helium. Science 322, 1525-1529 (2008).

3. Shafir, D. et al. Resolving the time when an electron exits a tunnelling barrier. Nature 485, 343-346 (2012).

4. Sokolovski, D. \& Baskin, L. Traversal time in quantum scattering. Phys. Rev. A 36, 4604-4611 (1987)

5. Landauer, R. \& Martin, T. Barrier interaction time in tunneling. Rev. Mod. Phys. 66, 217-228 (1994)

6. Keldysh, L. Ionization in the field of a strong electromagnetic wave. Sov. Phys JETP 20, 1307-1314 (1965)

7. Corkum, P. \& Krausz, F. Attosecond science. Nature Phys. 3, 381-387 (2007).
8. Baltuška, A. et al. Attosecond control of electronic processes by intense light fields. Nature 421, 611-615 (2003).

9. Fittinghoff, D. N., Bolton, P. R., Chang, B. \& Kulander, K. C. Observation of nonsequential double ionization of helium with optical tunneling. Phys. Rev. Lett. 69, 2642-2645 (1992).

10. Arissian, L. et al. Direct test of laser tunneling with electron momentum imaging. Phys. Rev. Lett. 105, 133002 (2010).

11. Serbinenko, V. \& Smirnova, O. Multidimensional high harmonic spectroscopy: A semi-classical perspective on measuring multielectron rearrangement upon ionization. J. Phys. B 46, 171001 (2013).

12. Litvinyuk, I. V. et al. Shakeup excitation during optical tunnel ionization. Phys. Rev. Lett. 94, 033003 (2005).

13. Bryan, W. et al. Atomic excitation during recollision-free ultrafast multi-electron tunnel ionization. Nature Phys. 2, 379-383 (2006).

14. Büttiker, M. \& Landauer, R. Traversal time for tunneling. Phys. Rev. Lett. 49, 1739-1742 (1982).

15. Dudovich, N. et al. Measuring and controlling the birth of attosecond XUV pulses. Nature Phys. 2, 781-786 (2006).

16. Steinberg, A. M., Kwiat, P. G. \& Chiao, R. Y. Measurement of the single-photon tunneling time. Phys. Rev. Lett. 71, 708-711 (1993).

17. Corkum, P. B. Plasma perspective on strong field multiphoton ionization. Phys. Rev. Lett. 71, 1994-1997 (1993).

18. Dahlström, J., L'Huillier, A. \& Mauritsson, J. Quantum mechanical approach to probing the birth of attosecond pulses using a two-colour field. J. Phys. B 44, 095602 (2011).

19. Lewenstein, M., Balcou, P., Ivanov, M. Y., L'Huillier, A. \& Corkum, P. B. Theory of high-harmonic generation by low-frequency laser fields. Phys. Rev. A 49, 2117-2132 (1994).

20. Salières, P. et al. Feynman's path-integral approach for intense-laser-atom interactions. Science 292, 902-905 (2001).

21. Soifer, H. et al. Spatio-spectral analysis of ionization times in high-harmonic generation. Chem. Phys. 414, 176-183 (2013).

22. Mairesse, Y. et al. Attosecond synchronization of high-harmonic soft X-rays. Science 302, 1540-1543 (2003).

23. Zhao, J. \& Lein, M. Determination of ionization and tunneling times in high-order harmonic generation. Phys. Rev. Lett. 111, 043901 (2013).

24. Torlina, L. \& Smirnova, O. Time-dependent analytical $R$-matrix approach for strong-field dynamics. II. One-electron systems. Phys. Rev. A 86, 043408 (2012)

25. Ivanov, M. Y., Spanner, M. \& Smirnova, O. Anatomy of strong field ionization. J. Mod. Opt. 52, 165-184 (2005).

26. Mairesse, Y. et al. High harmonic spectroscopy of multichannel dynamics in strong-field ionization. Phys. Rev. Lett. 104, 213601 (2010).

27. Rohringer, N. \& Santra, R. Multichannel coherence in strong-field ionization. Phys. Rev. A 79, 053402 (2009).

28. Smirnova, O. et al. High harmonic interferometry of multi-electron dynamics in molecules. Nature 460, 972-977 (2009).

29. Torlina, L., Ivanov, M., Walters, Z. B. \& Smirnova, O. Time-dependent analytical $R$-matrix approach for strong-field dynamics. II. Many-electron systems. Phys. Rev. A 86, 043409 (2012).

30. Weinkauf, R., Schlag, E., Martinez, T. \& Levine, R. Nonstationary electronic states and site-selective reactivity. J. Phys. Chem. A 101, 7702-7710 (1997).

\section{Acknowledgements}

The authors wish to thank O. Raz, J. M. Dahlström and M. Yu. Ivanov for valuable discussions. N.D. is the incumbent of the Robin Chemers Neustein Professorial Chair. N.D. acknowledges the Minerva Foundation, the Israeli Science Foundation, the Crown Center of Photonics and the European Research Council for financial support. N.D. and O.S. acknowledge the German-Israeli Foundation. L.T. and O.S. acknowledge the support of DFG grant SM 292/2-3. F.M. and O.S. acknowledge the support of the DFG grant SM 292/3-1. D.S.B. and O.S. acknowledge the support of Einstein foundation project A-211-55 Attosecond Electron Dynamics.

\section{Author contributions}

O.P., G.O., H.S., B.D.B. and A.J.U. performed the experiments. O.P., G.O., F.M., O.S. and N.D. analysed and interpreted the data. Simulations were implemented by O.P., V.S., A.G.H., D.S.B. and F.M. This project was supervised by N.D. and O.S. All authors discussed the results and wrote the paper.

\section{Additional information}

Supplementary information is available in the online version of the paper. Reprints and permissions information is available online at www.nature.com/reprints.

Correspondence and requests for materials should be addressed to O.P., O.S. or N.D.

\section{Competing financial interests}

The authors declare no competing financial interests. 


\section{Methods}

High harmonics are generated with $30 \mathrm{fs}, 1 \mathrm{kHz}, 800 \mathrm{~nm}$ laser pulses focused to deliver $2-4.5 \times 10^{14} \mathrm{~W} \mathrm{~cm}^{-2}$ peak intensity in a Helium $\left(I_{\mathrm{p}}=24.5 \mathrm{eV}\right)$ gas jet. We estimated $U_{\mathrm{p}}=26.5 \mathrm{eV}$ according to the harmonic cutoff. The SH field is produced using a $50 \mu \mathrm{m}$ type-I $\mathrm{BBO}\left(\beta-\mathrm{BaB}_{2} \mathrm{O}_{4}\right)$ crystal. The conversion ratio of the fields is below $1 \%$ to maintain the perturbative conditions of the experiment. The SH field is linearly polarized and initially perpendicular to the fundamental field. Group-velocity dispersion is compensated using a birefringent crystal (calcite). The subcycle delay of the SH field relative to the fundamental field is controlled using a pair of BK7 glass wedges. Paralle polarization is obtained using a $\lambda / 2$ zero-order waveplate for $800 \mathrm{~nm}$. High harmonics are generated by focusing the two beams into a pulsed $100 \mathrm{~Hz}$ gas jet. The harmonic spectrum is measured by means of an XUV spectrometer. 